Clinical Medicine

Abstract ID: 118

\title{
Procalcitonin and Interleukin-6 for predicting culture- negative and culture positive bacterial sepsis in critically ill patients with systemic inflammatory response syndrome
}

\author{
Mohd Basri Mat Nor I Azrina Md Ralib \\ Department of Anaesthesiology and Intensive Care, Kulliyyah of Medicine, International Islamic \\ University Malaysia
}

Introduction: Differentiation between culture-negative bacterial sepsis (BS), culturepositive BS and non-infectious systemic inflammatory response syndrome (SIRS) among critically ill patients remains a diagnostic challenge to the intensive care unit (ICU) physicians. This study aimed to evaluate the role of procalcitonin (PCT) and interleukin-6 (IL-6) in predicting non-infectious SIRS, culture-negative BS and culture-positive BS in the ICU. Methods: This prospective observational study was conducted in a tertiary ICU in Pahang. The patients were divided into sepsis and non-infectious SIRS based on clinical assessment with or without positive cultures. Patients with positive cultures were further divided into bacteraemia and positive other culture. The PCT and IL-6 were measured daily over the first 3 days. Results: Two hundred and thirty nine consecutive patients diagnosed with SIRS were recruited, of whom 164 (69\%) had sepsis. Among sepsis patients, there were $62(37.8 \%)$ culture positive and $102(62.2 \%)$ culture negative. Of these, 27 (16.5\%) develop bacteraemia. The most common site of infection was respiratory $(34.4 \%)$. Post-LSD analyses showed significant difference in the PCT between culture negative sepsis and SIRS ( $p=0.01)$; and positive other culture and SIRS $(p=0.04)$. On the other hand IL- 6 cannot differentiate between SIRS and negative culture sepsis $(p=0.06)$. Both PCT and IL-6 predicted bacteraemia with an AUC of 0.70 ( 0.57 to 0.82$)$ and 0.68 (0.53 to 0.70$)$. IL-6 is independently associated with bacteraemia and other culture after adjusting for age, sex, hypertension, SAPS II score and day 1 PCT. Conclusions: Procalcitonin but not Interleukin-6 is able to differentiate SIRS from culture-negative BS. However, IL-6 is independently associated with bacteraemia and other culture.

KEYWORDS: Procalcitonin, Interleukin-6, culture-negative sepsis, SIRS 